\title{
BOREHOLES PLANS OPTIMIZATION METHODOLOGY COMBINING GEOSTATISTICAL SIMULATION AND SIMULATED ANNEALING
}

\author{
Marisa Pinheiro ${ }^{1}$, Xavier Emery ${ }^{2,3}$, Ana Maria A. C. Rocha ${ }^{4}$, Tiago Miranda ${ }^{1}$, Luís \\ Lamas $^{5}$
}

\begin{abstract}
Nowadays, the prospection plans have the difficult task of ensuring a more complete and rich characterization of the rock mass for the purpose of optimizing costs and increasing safety in geotechnical projects. Currently, boreholes location and depth are mainly defined based on experience and know-how of professionals, as such, it is userdependent. Hence, there is a lack of methodologies to help the decision-makers in defining the optimal location of boreholes (with relevant information). Therefore, this paper presents a methodology based on the use of geostatistical conditional simulation allied to a stochastic global optimization algorithm (Simulated Annealing) to develop optimized boreholes plans comparing a uni-objetive and a multi-criteria optimization approaches. In this work, the optimized location is considered the one that minimizes uncertainty translated by either the average local variance or the average width of $95 \%$ probability intervals of simulated values at unsampled locations. This methodology was applied using preliminary information obtained from previously executed boreholes using as variable the empirical rock mass classification system, Rock Mass Rating, in a Chilean deposit.
\end{abstract}

Keywords: Geostatistical Simulation; Boreholes optimization; Simulated Annealing; Multi-criteria approach; Geotechnical prospection plans.

\footnotetext{
${ }^{1}$ ISISE, University of Minho, Azurém, 4800-058, Guimarães, Portugal

${ }^{2}$ Department of Mining Engineering, University of Chile, Avenida Tupper 2069, Santiago, Chile

${ }^{3}$ Advanced Mining Technology Center, University of Chile, Avenida Beauchef 850, Santiago, Chile

${ }^{4}$ Algoritmi Research Centre, University of Minho, Azurém, 4800-058, Guimarães, Portugal

${ }^{5}$ Portuguese National Laboratory for Civil Engineering - LNEC, Lisboa, Portugal
} 


\section{INTRODUCTION}

Rock mass prospection, mainly regarding boreholes, involves very high costs. Moreover, due to the frequently large spacing between boreholes and the fragmentary nature of the obtained data, considerable uncertainties affect the geotechnical models, mostly in highly heterogeneous rock masses. Currently, boreholes location and depth are mainly defined based on experience and know-how of professionals, as such, it is user-dependent. Therefore, the search for more rational ways of planning the borehole locations, as they can provide higher quality data and decrease the uncertainties, is of utmost importance, essentially in large geotechnical projects.

Usually, the time and money available for rock mass model construction is very short. The geotechnical prospection plans in large geotechnical works are generally divided into two phases: the initial phase where a preliminary and confined characterization is carried out, and a second phase where the number of executed boreholes, as well as laboratory and in situ tests, are significantly higher. Thus, the proposed methodology can be applied in the second phase, using the preliminary information obtained from the initial phase. This methodology intends to fill the existing gap of consolidated methodologies for this purpose, and to help professionals to optimize the boreholes position in the second phase of the prospection works by giving them information regarding the borehole quantity and depth.

In this search, a few existing methodologies for boreholes optimization combining different types of algorithms, in which the goal consists in minimizing a wide range of uncertainty measures obtained by using geostatistical techniques, were found. In detail, McBratney et al. (1981), Scheck and Chou (1983) and Olea (1984) presented methodologies to minimize the sampling requirements necessary to predict a regionalized variable at a specific level of accuracy based on the maximum or on the average standard kriging error as a global index of sampling efficiency. Subsequently, Englund and Heravi (1994) applied such a methodology to assess the number of samples and sampling phases required to remediate a contaminated soil balanced with reasonable costs. Marchant and Lark (2006) developed an approach to optimize the sampling scheme used to identify the spatial continuity (variogram) of the variable of interest. The goal was to understand what type of sampling scheme could result in more accurate variograms to use in further simulations and, consequently, reduce the 
sampling costs. In each phase the information from previous phases is used to generate new information and to decide if a new phase is required. As an objective function, the authors minimized the developed expression to evaluate the uncertainty of variogram parameter estimation (sill, range, nugget, etc.). Van Groenigen et al. (1999, 2000) and Brus and Heuvelink (2007) presented more complex methodologies using the Simulated Annealing optimization algorithm to find the pattern for new samples that minimizes the average kriging variance. Soltani and Hezarkhani $(2009,2013$ b) also proposed a simulated annealing methodology, which aims to maximize the kriging variance reduction, calculated after dividing the kriging variance obtained from the initial samples with the kriging variance obtained with new additional boreholes. The same authors published a related work (Soltani and Hezarkhani, 2013a), this time combining the simulated annealing algorithm and an objective function to assess the value information that additional boreholes will bring for the deposit characterization, based on the range of reliability of each individual block resulting from its prediction. Similarly, Soltani et al. (2011) proposed the use of a genetic algorithm instead of simulated annealing, and the average kriging variance as the objective function to minimize.

These statements lead to the identification of a limitation in optimal boreholes sampling strategies, mostly because the use of kriging only results in a single outcome for the random field and the obtained uncertainty metrics (kriging variance and related metrics) do not reflect the local variability of the regionalized variable under consideration, such as proportional and regressive effects, i.e., a local dispersion that depends on the local mean value (Chilès and Delfiner, 2012). Thus, the replacement of kriging by geostatistical simulation that results in several outcomes (realizations) and, consequently, in a greater uncertainty reduction and accuracy in the spatial variability quantification of a random field, is a way to overcome the aforementioned limitation.

As previously mentioned, the proposed methodology will work as a helping tool in supporting the decision-maker when defining the prospection plans. To establish this methodology, it is necessary to combine two important techniques: the geostatistical simulation of the geotechnical variable of interest, conditionally to the available preliminary information, and an optimization algorithm known as simulated annealing (SA). The methodology can be divided into three major steps: first, preliminary 
information, i.e. geotechnical information resulting from preliminary boreholes, is required; second, the data should allow performing geostatistical simulation to obtain the objective functions needed in the optimization process; and the third and last step culminates by performing the optimization with SA. As a result of this optimization, it is possible to extract the optimal position for additional boreholes, the gain in terms of geotechnical details and the minimum depth of each borehole. It is worth mentioning that the methodology is of easy use and presents considerably low pre- and postprocessing times.

The paper is organized as follows. The general global optimization algorithm and the developed methodology to optimize boreholes plans are presented in Sections 2 and 3, respectively. A case study is then described in Section 4, with a short introduction of the data and a presentation of the results of geostatistical simulation. In Section 5, the optimization results are presented considering uni- and multi-criteria approaches. Discussion and conclusions follow.

\section{SIMULATED ANNEALING}

In the metallurgical industry, a thermal process named annealing aims to forge iron in order to minimize the energy spent to cool and freeze the metal. Then, the metal is heated to a maximum temperature able to change its physical properties (creating a particle disorder) and followed by slow cooling to guarantee that the final configuration of the solid is structurally superior. Simulated annealing (SA) (Kirkpatrick et al., 1983) is an iterative algorithm to solve combinatorial optimization problems inspired in this process in order to find a balanced state for each temperature, this way minimizing the internal energy of the process. In engineering problems, the use of SA has been increasing once it is an alternative to gradient-based methods or other local classical methods that can be trapped in local optima.

This algorithm starts by randomly generating two solutions at each iteration, the socalled new solution $(j)$ that emerges after random changes in the parameters that generate the previous solution $(i)$. Then, in the case of facing a minimization problem, SA compares the objective function (OF) values for each solution. On the one hand, if $O F_{j} \leq O F_{i}$, solution $j$ is automatically accepted and assumed as the temporary best 
solution. Under these conditions, the algorithm jumps to another iteration and new solutions are generated. On the other hand, if $O F_{j} \leq O F_{i}$ there is a possibility to accept solution $j$, even if it is a "worse" solution than solution $i$. By allowing these controlled uphill moves to counter the downhill moves, the algorithm is forced towards the global minimum that sometimes can be found near the worst solutions. This selection is made through the calculation of an acceptance probability $\left(P_{\text {accept }}\right)$ that depends on a temperature parameter that decreases in a slow rhythm to avoid, once again, the algorithm to be trapped into a local minimum:

$$
P_{\text {accept }}= \begin{cases}1 & O F_{j} \leq O F_{i} \\ e^{\frac{-\Delta O}{T}} & \text { otherwise }\end{cases}
$$

wherein $\Delta O$ represents the difference between the $O F$ values in the states $i$ and $j(\Delta O=$ $\left.O F_{j}-O F_{i}\right)$ and $T$ is the control temperature. In the case of lower temperature values, the probability of accepting worse solutions is also lower, allowing SA to converge faster.

As previously mentioned, the initial temperature to start the process must be high enough to allow SA exploring all the space of solutions. However, during the process, the temperature is progressively reduced until a threshold value defined by the user. This cooling should be slow in order to avoid rushing the stopping criteria of the algorithm, e.g.:

$$
T_{j}=\alpha \times T_{i}
$$

where $T_{i}$ represents the temperature value assumed when solution $i$ is generated and $\alpha$ represents the cooling constant, whose value ranges from 0.70 to 0.99 for a fast and slow cooling, respectively (Aarts and Korst, 1989).

Besides the previously mentioned parameters, others should be defined:

- A perturbation or transition kernel, which indicates the mechanism used to generate a new solution to be tested given a current solution.

A maximum number of allowed moves for each temperature value. This number translates the number of times that SA generates new solutions before decreasing the temperature. Once reached this maximum number of moves, the temperature is 
decreased using the aforementioned cooling process. Additionally, the maximum number of accepted solutions given the same temperature value must also be defined.

- A stopping criterion: this criterion can be defined based on one or more key parameters. Many authors stop SA by defining a final value for the temperature, while others add more criteria to the process (Yang, 2010; Brus and Heuvelink, 2007; Hernandez and Emery, 2009). In the present case, the stopping criterion will be composed by a temperature, an iteration number and a maximum number of rejections within a given temperature state. The latter will allow stopping the algorithm if no progress is shown.

To sum up, the algorithm should be applied using the following general steps:

Step 1: The values of the above key parameters (initial temperature, maximum of iterations, cooling constant and maximum number of rejections in a given temperature state) must be set. This can be defined after performing a sensitivity analysis to identify which parameter values allow the convergence of the algorithm to the global minima, or using as a reference existing values for similar optimization problems.

Step 2: In the first iteration, an initial solution $i$ should be randomly generated within the space limits. For the remnant process, the new generated solutions are based in random changes made to the current solution.

Step 3: The objective function is calculated for the new solution $j$ and the decision of whether or not moving to the new solution is made.

Step 4: The temperature is decreased until reaching the thresholds defined for the stopping criterion, for which the algorithm gives the near-optimal solution for the optimization problem. 


\section{PROPOSED METHODOLOGY}

\subsection{Overview}

In spite of the decreasing uncertainty associated with the rock mass geotechnical model, as the number of executed boreholes increases, it seems essential to optimize the number and cost of the boreholes in the prospection plans, as the location and depth of these boreholes considerably influence the quality of the characterization model. As such, it is imperative to accomplish a trade-off between the geotechnical detail and the number of necessary boreholes, and the proposed methodology allows precisely that. It gives not only the adjustment of the minimum number of boreholes to execute as a complement of preliminary boreholes, resulting in a prospection plan with a certain quality, but also the optimal positions of these boreholes through the minimization of the uncertainty associated with the geotechnical model.

In detail, the methodology starts by performing a geostatistical simulation from preliminary data (input), for which a wide range of geotechnical information can be obtained such as the Fracturing Degree (F), Weathering Degree (W), Rock Quality Design (RQD) or the empirical rock mass classification systems, like Rock Mass Rating (RMR) (Bieniawski, 1989), Q-system (Barton, 1974) or Geological Strength Index (GSI) (Hoek, 1994). Geostatistical simulation is performed in a conditional way, thus guaranteeing that the preliminary data are reproduced in information and position, following the methodology presented by Pinheiro et al. (2016) (Fig. 1: Stage 1). As a result, a total of $L$ realizations of the geotechnical information, which enable the determination of the objective function to use in the optimization process, are obtained. In this case, two metrics will be used as objective functions: the local variance of the simulated values and the width of their $95 \%$ probability interval. Then, the SA algorithm is used to minimize the objective function values, which translates the uncertainty of the geotechnical model. In this sense, the output of the previously explained process can obtain the optimal length and position of additional boreholes to execute during the second phase of the prospection plans (Fig. 1: Stage 2). 


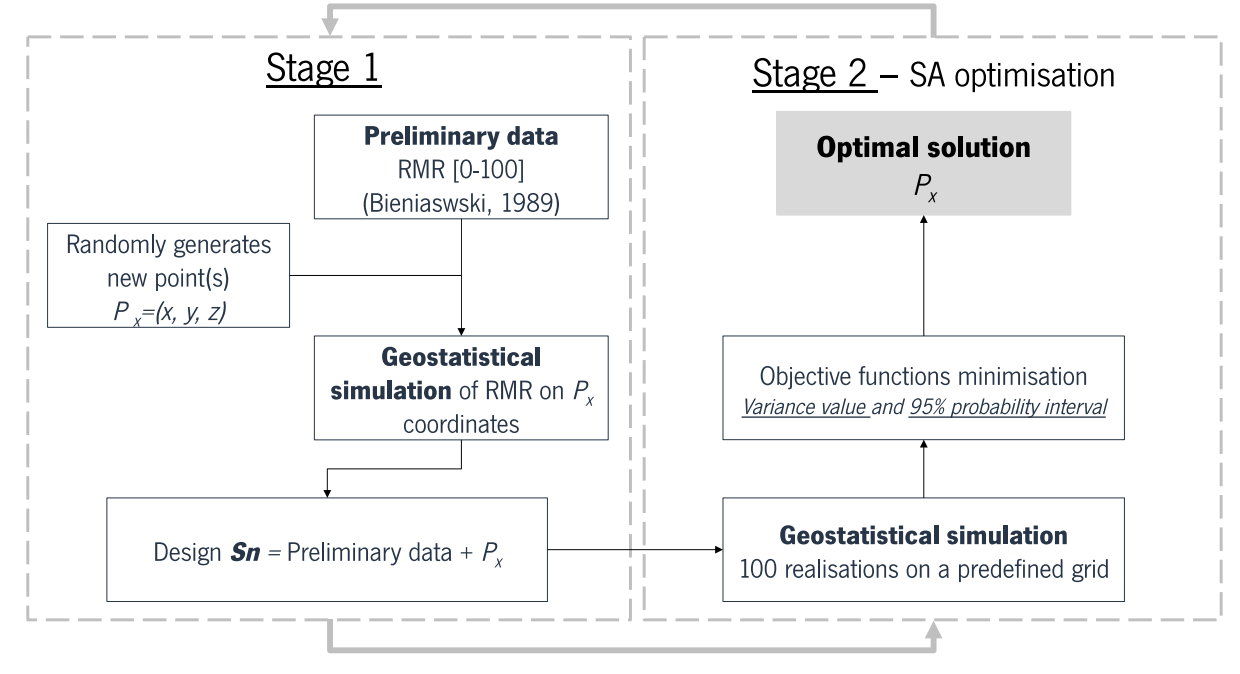

Fig. 1. Scheme of the proposed methodology combining geostatistical simulation and simulated annealing.

\subsection{Optimization process}

As already mentioned, the methodology requires geotechnical information to use as preliminary data. In general, this information is obtained from a limited number of boreholes already executed in the field, the location and depth of which are usually based on the know-how of the professionals and geological aspects. This geotechnical information should allow the calculation and fitting of a variogram of the measured regionalized variable (Chilès and Delfiner, 2012), which is an imperative tool to use in geostatistical simulation. The type of information used as initial data in this optimization process can be diverse and directly derived from the borehole data. Note that there is no deterministic number of preliminary information to use in order to apply the methodology, it should be enough to allow the experimental variogram computation that depends on the variable spatial behavior.

In what concerns the optimization process, it is initialized by randomly generating $n$ points in a confined space used to represent the additional boreholes to execute. Then, conditional simulation is performed and a total of $L$ realizations of the chosen variable at the $n$ target points are obtained. At this stage, the turning bands algorithm is used to construct the realizations and the residual kriging approach is used to condition the realizations to the preliminary data values, i.e. to force the realizations to honor the 
information and position of the preliminary data (Emery and Lantuéjoul, 2006). The average of the $L$ realizations is computed in order to represent each target point with a single value. After this process, each additional point has some simulated information comparable to the preliminary data and the remaining optimization process can then be executed. The purpose of this first stage is to investigate the contribution of each point in reducing the uncertainty of the geotechnical model by using the simulated values.

In the second stage and using the $L$ realizations average values of each $n$ simulated point assigned to the additional boreholes to execute, are joined to the preliminary data, resulting in a new sampling design $(S n)$. Consequently, using the points of the new design, once again a conditional simulation is executed, this time using as target points a predefined grid $(2 D$ or $3 D)$ covering the region of interest. As an outcome of this simulation process, the objective function to be minimized in order to find out the optimal solution for the presented problem is obtained (Fig. 2). All the geostatistical routines and the SA algorithm have been programmed in a Matlab environment (Emery and Lantuéjoul, 2006; Lin and Fei, 2012; Yang, 2010), using a desktop computer with an Intel ® Core TM i7-3610QM CPU @2.30GHz processor.

Taking into account that each borehole lists information at several depth ranges, i.e. each borehole contains the geotechnical information at several points, the optimization is also made by considering alignments of points in the generation of the sampling design. Therefore, in this work, the developed methodology is also tested by assuming each borehole as a vertical alignment of points originated from an isolated point in the surface (header). This action allows the integration of all vertical information that can emerge from a borehole and best represents the reality of them. 


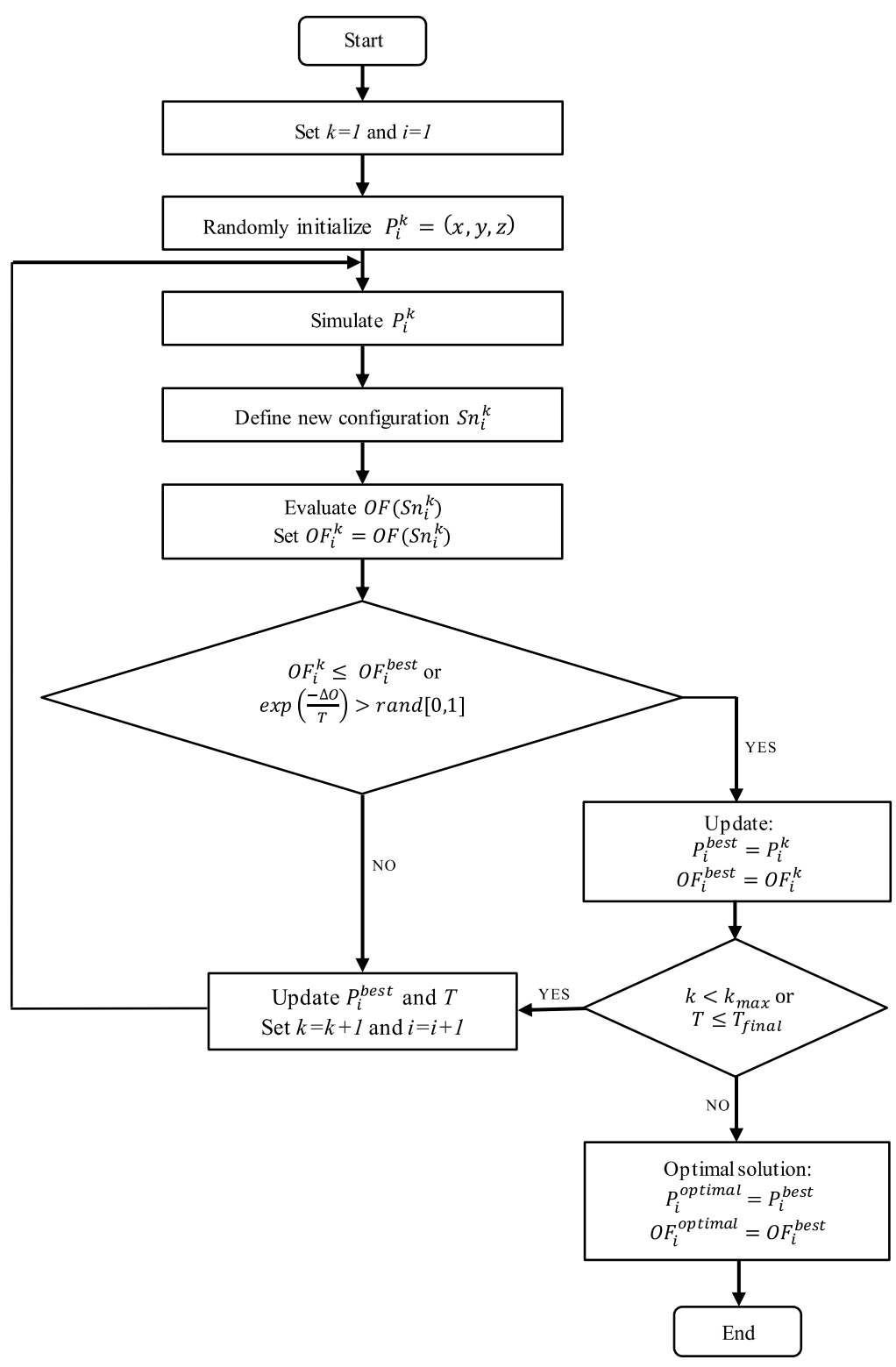

Fig. 2. Simulated annealing workflow adapted to the proposed methodology.

\subsection{Uni-objective approach}

The bound constrained problem to be addressed here has the following form:

$$
\begin{array}{cc}
\min & O F(S n) \\
\text { subject to } & l b \leq S n \leq u b
\end{array}
$$

where $O F(S n)$ represents the value of the objective function for design $S n$. The bound constraints on the values of the variables $S n$ are related to the rock block dimensions, 
where the lower $(l b)$ and upper $(u b)$ bounds give the minimum and maximum values of the $(x, y, z)$ coordinates, respectively.

Thereafter two distinct objective functions are considered in the optimization process: the variance of simulated values and the width of the $95 \%$ probability intervals of the simulated values. These two metrics are considered in order to provide more options to the decision-maker in choosing the metric that, from his/her point of view, is able to better represent the uncertainty of the geotechnical model. To simplify the notation, the argument of each objective function will be shown in the following sections.

The first objective function to be presented is the average width of the probability intervals obtained using the $L$ realizations results, calculated as follows:

$$
\overline{P I}_{95 \%}=\operatorname{mean}\left(P I_{95 \%}\right)
$$

where $P I_{95 \%}$ is obtained for each target location from the set of $L$ simulated values at this location, by calculating the difference between the percentiles of an inferior limit and a superior limit given by $(1-p) / 2$ and $(1+p) / 2$, respectively, for a probability $p$ equal to 0.95 . After that, the average of the $\overline{P I}_{95 \%}$ widths over all the target points is computed and used as an objective function. The $\overline{P I}_{95 \%}$ final value should be as low as possible to decrease the uncertainty associated with the geotechnical property at unsampled locations.

The second objective function is based on the variance of the simulated values for each created design $(S n)$, calculated after the execution of two main steps: first, the variance of the $L$ simulated values is calculated at each location; second, the average value of these variances over all the target locations is computed. Again, the lower this average variance, the lower the uncertainty at unsampled locations.

Table 1 shows details of the first step, where $N$ represents the total number of grid nodes that compose each $S n$ design, $L$ is the total number of geostatistical realizations, and $X_{p, L}$ represents the simulated value for point $p$ at realization $L$. 
Table 1 First step to calculate the variance value.

\begin{tabular}{ccccc}
\hline Sn points & \multicolumn{3}{c}{ Realization number } & Local variance \\
\hline 1 & 1 & 2 & $L$ & $\operatorname{Var}_{1}=\frac{1}{L} \sum_{l=1}^{L}\left(X_{1, l}-\bar{X}_{1}\right)^{2}$ \\
2 & $X_{1,1}$ & $X_{1,2}$ & $X_{1, L}$ & $\operatorname{Var}_{2}=\frac{1}{L} \sum_{l=1}^{L}\left(X_{2, l}-\bar{X}_{2}\right)^{2}$ \\
$N$ & $X_{2,1}$ & $X_{2,2}$ & $X_{2, L}$ & \\
& $X_{N, 1}$ & $X_{N, 2}$ & $X_{N, L}$ & $\operatorname{Var}_{N}=\frac{1}{L} \sum_{l=1}^{L}\left(X_{N, l}-\bar{X}_{N}\right)^{2}$ \\
\hline
\end{tabular}

Thus, the objective function based on the variance is given by,

$$
\operatorname{Var}=\frac{1}{N} \sum_{p=1}^{N} \operatorname{Var}_{p}
$$

\subsection{Multi-criteria approach}

Taking into account the difficulty and plurality of possibilities associated with in situ decisions in geotechnics, a multi-criteria approach is also considered in this work. The goal is to facilitate future decisions and to analyze the effectiveness of the objectives (used metrics) when applying SA to boreholes optimization.

Therefore, the two previously presented objective functions (criteria) are combined into a single function, since they can be complementary. Using this multi-criteria approach, the decision-maker will be able to use both objectives (metrics) and to identify one criterion that is dominated by the other by analyzing the trade-off existing between them (Kalamaras et al., 2000).

Hence, the multi-criteria problem to be optimized exhibits the following form:

$$
\begin{array}{cc}
\min & \left(O F_{1}(S n), O F_{2}(S n)\right) \\
\text { subject to } & l b \leq S n \leq u b
\end{array}
$$

where,

$$
O F_{1}=\frac{\overline{P I}_{95 \%}}{\overline{P I}_{95 \%, \text { initial }}}
$$




$$
O F_{2}=\frac{\text { Var }}{\text { Varinitial }_{\text {in }}}
$$

Note that $O F_{1}$ and $O F_{2}$ are the objective functions normalized using the values of the objective functions of the preliminary data (initial data used for conditioning the geostatistical simulation). As mentioned before, the argument $S n$ is removed in order to simplify the notation. $\overline{P I}_{95 \%}$ represents the average width of the $95 \%$ probability intervals and $\operatorname{Var}$ is the average variance obtained after the geostatistical simulation of the tested design $(S n) . \overline{P I}_{95 \% \text {,initial }}$ and Var $_{\text {initial }}$ denote the average width of the $95 \%$ probability intervals and the average variance, respectively, obtained after geostatistical simulation on the predefined grid conditioned only to the preliminary data.

The Weight Sum Method (Zadeh, 1963) is a classical multi-objective method that allows standardizing a set of objectives into a single objective function by multiplying each one by a weight. This method is chosen due to its simple use, as well as the assurance in finding the ideal and admissible space of solutions (Marler and Arora, 2009). The weight definition can be made using different methods. However, in this study the weights are attributed manually since the weight vector and the solution vector do not show a linear correspondence. The manual weight definition intends to simulate the decision-maker perspective, regarding the objective function. Hence, the problem to be minimized is converted to a uni-objective problem by aggregating the two objective functions into a single one, given by:

$$
\begin{array}{cc}
\min & \left(W_{1} \cdot O F_{1}(S n)+W_{2} \cdot O F_{2}(S n)\right) \\
\text { subject to } & W_{1}+W_{2}=1 \\
& l b \leq S n \leq u b
\end{array}
$$

where $W_{1}$ is the weight for objective function $O F_{1}$ and $W_{2}$ is the weight for objective function $O F_{2}$.

\section{CASE STUDY}

\subsection{Presentation}

The proposed methodology is applied using information of mechanical boreholes from an epithermal gold deposit located in the Cordillera de Los Andes, region of Atacama, 
northern Chile. The geotechnical information gathered from the boreholes is the empirical Rock Mass Rating (RMR) system. Regarding the regional geology of the area, it is characterized by a group of intrusive, volcanic and sedimentary rocks, affected by fault zones that control the mineralization.

Considering the high quantity of the available data it is necessary to restrict the information to a confined area (block). The optimization is applied to two different scenarios, one using isolated points, and the second one using vertical alignments of points that better represent the reality of boreholes. In what concerns the scenario with isolated points, a total of 22 points are selected from the available boreholes to represent the preliminary information to use in the geostatistical simulation and in the optimization procedure. Regarding the scenario with vertical alignments of points, a total of 6 boreholes are chosen as the preliminary information and each alignment to be found will be composed by 12 points evenly spaced along the depth.

Each one of the 22 points and 6 boreholes contains information about the RMR within a rock block with the dimensions of $120 \mathrm{~m} \times 440 \mathrm{~m} \times 220 \mathrm{~m}$ along the east $(X)$, north $(Y)$ and vertical $(Z)$ directions. Fig. 3 maps the 22 points allowing the visualization of the preliminary data, while Fig. 4 presents the 6 chosen alignments.

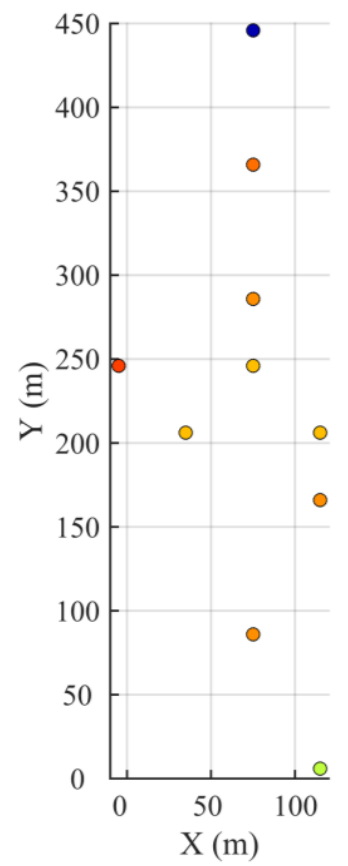

(a)

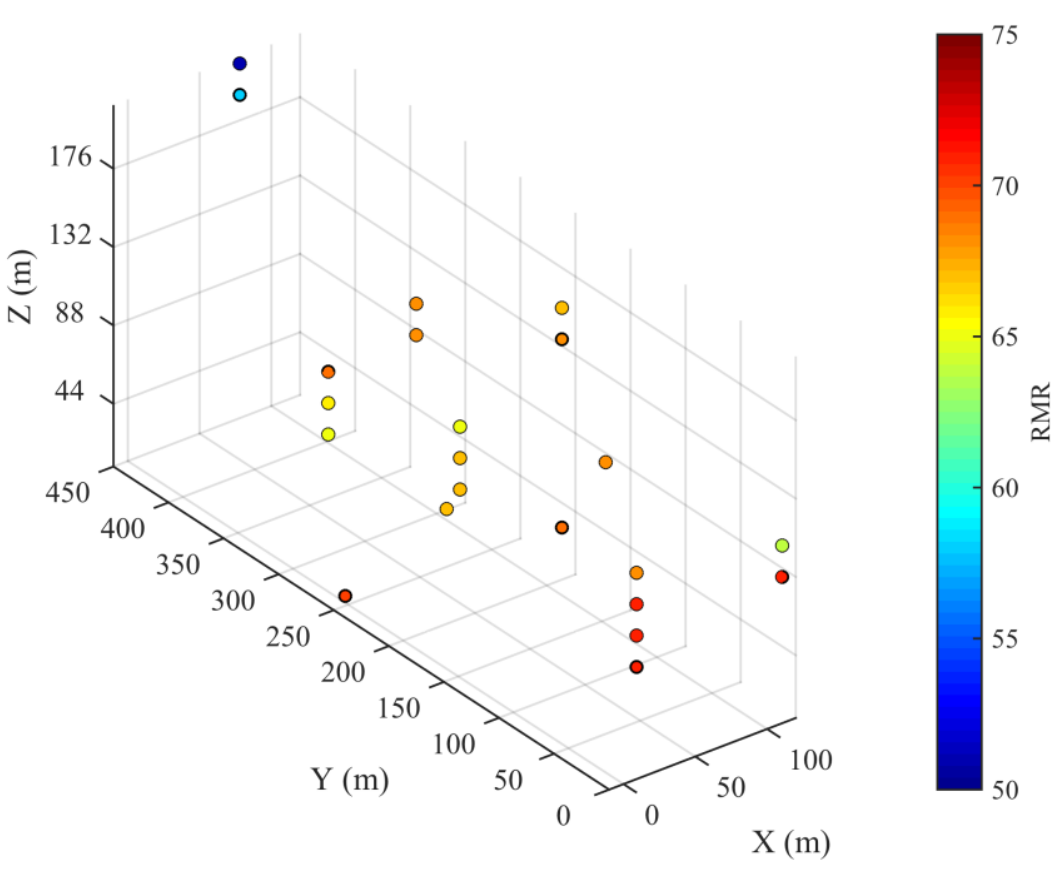

(b) 
Fig. 3. Mapping of the initial boreholes data used in the isolated points scenario in: a) $X Y$ plane; and b) $X Y Z$ perspective $(X, Y$ and $Z$ in meters).

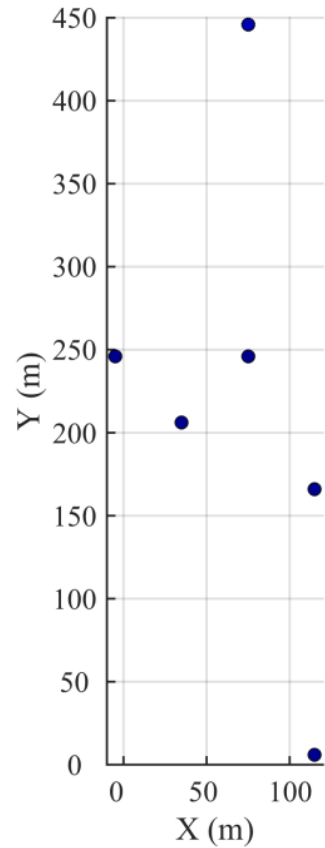

(a)

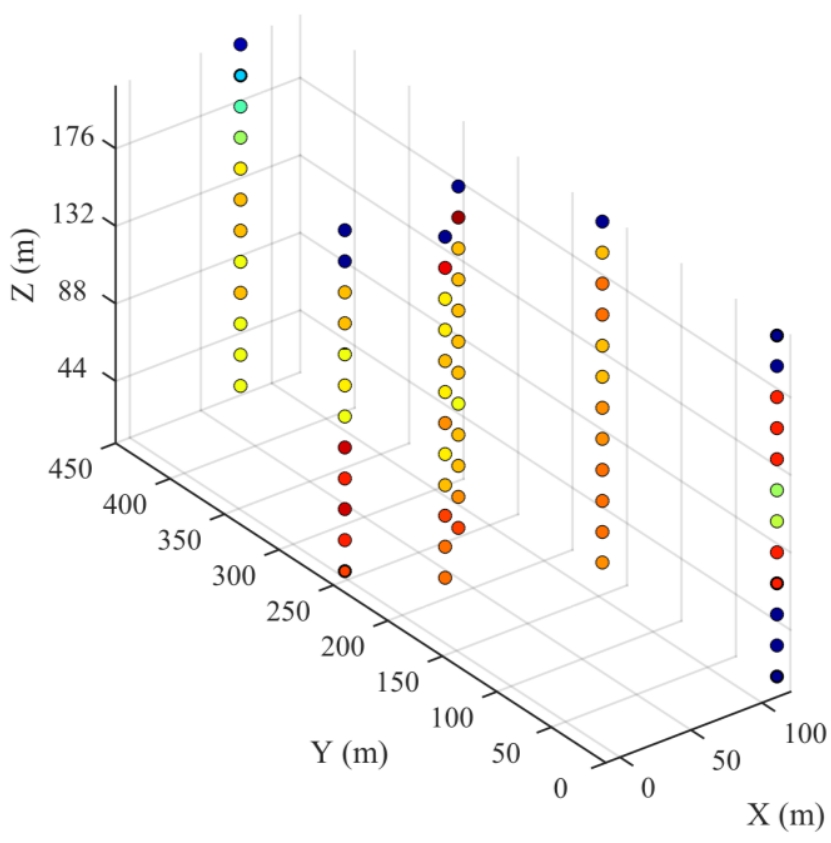

(b)

Fig. 4. Mapping of the initial boreholes data used in the vertical alignments scenario in: a) $X Y$ plane; and b) $X Y Z$ perspective ( $X, Y$ and $Z$ in meters).

\subsection{Geostatistical simulation}

Regarding the geostatistical simulation conditioned to the preliminary data, some steps proposed by Pinheiro et al. (2016a, 2016b) need to be implemented:

1) First, the RMR preliminary data are analyzed through the calculation of basic statistics (Table 2). According to these statistics, the rock mass in consideration shows a good quality, insofar as the RMR value ranges from 51 to 71, with a mean of 67 that can classify the rock mass as almost homogeneous.

2) The preliminary data corresponding to the 22 points or to the 6 alignments are then transformed into data with a standard Gaussian distribution with a zero mean and a unit variance. Such a transformation is necessary for subsequent geostatistical modeling and simulation (Chilès and Delfiner, 2012).

3) The experimental variograms of the Gaussian data are computed, using either the isolated points or the point alignments. These variograms are computed until a 
maximum distance of $100 \mathrm{~m}$, beyond which the data values are found to exhibit a low correlation.

4) The experimental variograms are fitted using isotropic spherical functions, as presented in Equations (10) and (11), where the distance written between brackets represents the correlation range and the number before the spherical structure denotes the adopted value for the sill (Chilès and Delfiner, 2012):

Scenario with isolated points:

$$
\gamma=0.495 \text { Spherical }(40 \mathrm{~m})
$$

Scenario with point alignments:

$$
\gamma=0.80 \text { Spherical }(70 \mathrm{~m})
$$

5) A two-dimensional regular grid with a mesh of $5 \mathrm{~m} \times 5 \mathrm{~m}$ and a total of 60 nodes along the east direction and 120 nodes along the north direction is defined to conditionally simulate the RMR and to calculate the objective functions.

6) A Gaussian random field is simulated at the target grid nodes using the turning bands method (Emery and Lantuéjoul, 2006; Pinheiro et al., 2016a, 2016b). The number of turning lines used to generate the random field is 1500 , while the number of realizations is set to $L=100$, so that the post-processing outputs (variances and probability intervals) can be calculated with a reasonable approximation (this number of realizations is commonly used in geostatistical applications and is suggested by Chilès and Delfiner (2012)). Residual kriging is then used to condition the simulation to the preliminary data, with a unique neighborhood implementation.

7) The simulated Gaussian values for each realization are finally back-transformed into their original scale (RMR).

Table 2 Basic statistics on RMR preliminary data and their Gaussian transforms.

\begin{tabular}{lcccc}
\hline & \multicolumn{2}{c}{ 22 isolated points } & \multicolumn{2}{c}{ 6 point alignments } \\
\cline { 2 - 5 } & $R M R$ & Gaussian values & RMR & Gaussian values \\
\hline Number of points & 22 & 22 & 62 & 62 \\
Mean & 66.77 & 0.00 & 67.29 & 0.00 \\
Variance & 19.99 & 0.94 & 12.27 & 0.98 \\
Minimum & 51.00 & -2.00 & 51.00 & -2.41 \\
\hline
\end{tabular}




\begin{tabular}{lllll}
\hline Maximum & 71.00 & 2.00 & 74.00 & 2.41 \\
\hline
\end{tabular}

To understand the differences between the preliminary data and the geotechnical improvements when new boreholes are added, the simulation conditioned only to the preliminary data is also performed on the predefined grid. As a result, the values obtained for the variance and width of $95 \%$ probability interval objective functions are Var $_{\text {initial }}=9.00$ and $\overline{P I}_{95 \% \text {,initial }}=11.28$ when using the 22 isolated points and Var $_{\text {initial }}=9.39$ and $\overline{P I}_{95 \% \text {,initial }}=11.36$ when using the 6 point alignments. As an example, Fig. 5 shows the first realization of the RMR and the variance of 100 realizations, conditioned to the preliminary data. Regarding the simulation computational time, it can be divided in two: 1) the time required to construct 100 realizations on the randomly generated coordinates, which was approximately $30 \mathrm{~min}$; and 2) the time required to construct 100 realizations of the new design configuration $S n$ on the target grid, which takes more or less $1 \mathrm{~h}$.

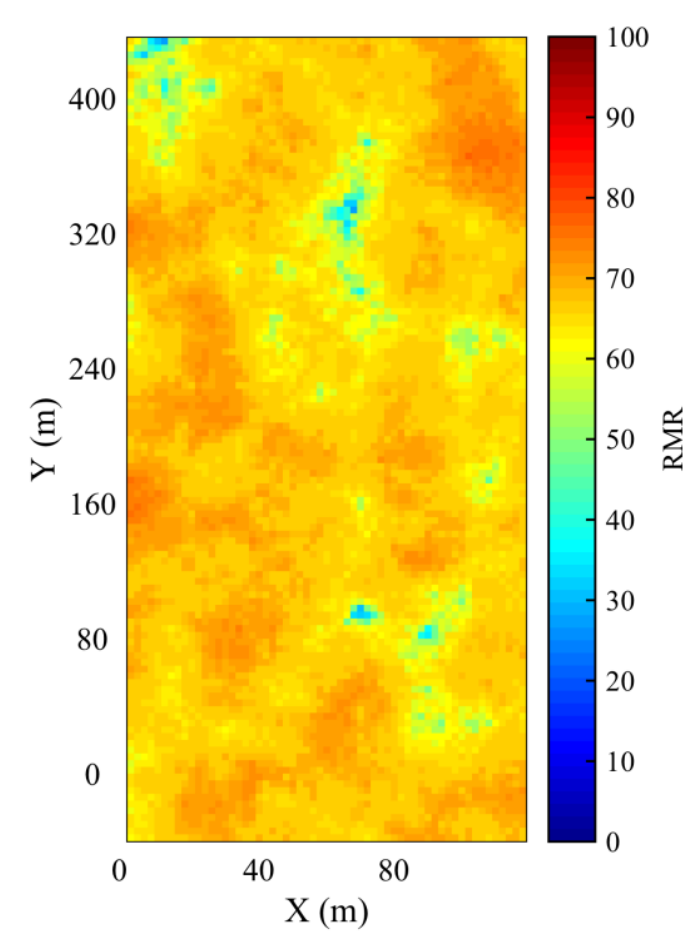

(a)

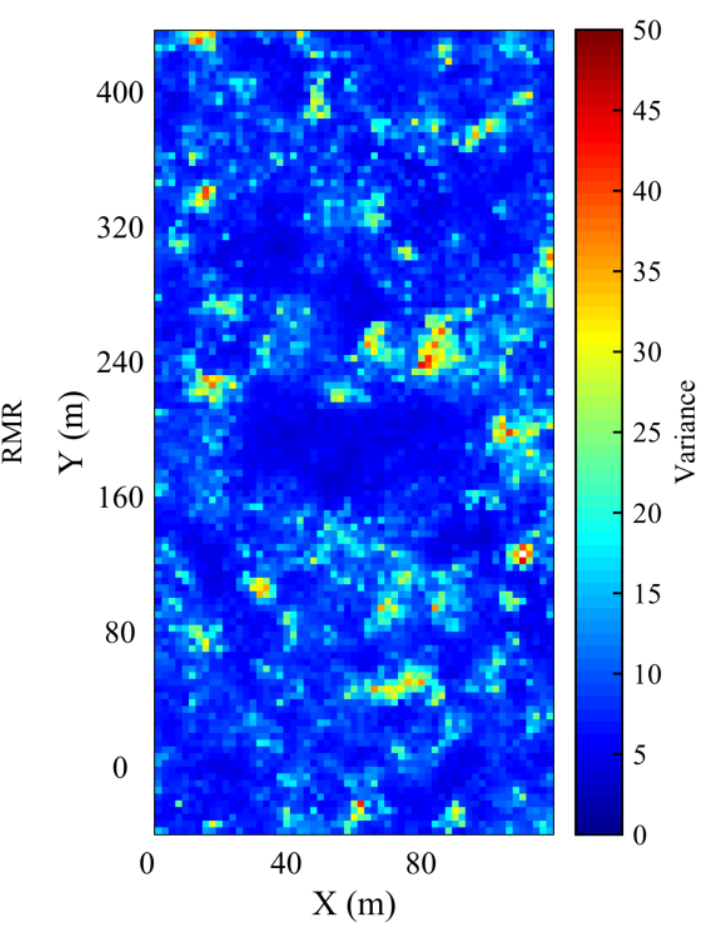

(b)

Fig. 5. RMR preliminary data geostatistical simulation results for: a) first realization; b) variance of 100 realizations 


\section{OPTIMIZATION RESULTS}

In order to execute the SA and to start the optimization process, some internal parameters need to be defined. Therefore, a sensitivity study is carried out, which enables to understand the influence of each parameter and find the best values for the algorithm. As such, the internal values adopted in this optimization are shown in Table 3.

Table 3 SA internal parameters.

\begin{tabular}{cccccc}
\hline $\begin{array}{c}\text { Cooling } \\
\text { factor }\end{array}$ & $\begin{array}{c}\text { Initial } \\
\text { temperature } \\
\left({ }^{\circ}\right)\end{array}$ & $\begin{array}{c}\text { Final } \\
\text { temperature } \\
\left({ }^{\circ}\right)\end{array}$ & $\begin{array}{c}\text { Maximum } \\
\text { number of } \\
\text { rejections }\end{array}$ & $\begin{array}{c}\text { Maximum } \\
\text { number of } \\
\text { moves }\end{array}$ & $\begin{array}{c}\text { Maximum } \\
\text { number of } \\
\text { acceptances }\end{array}$ \\
\hline 0.80 & 2.00 & 0.01 & 100 & 50 & 10 \\
\hline
\end{tabular}

\subsection{Uni-objective results}

Regarding the validation of the proposed optimization methodology, a wide range of additional points and alignments of points are considered.

\subsubsection{Isolated point optimization}

The point optimization process starts by adding one point to the preliminary 22 points, so $n=1$, and then by adding points consecutively one by one up to a total of 13 additional points. A number of points of 16, 20, 24 and 30 are also tested in order to accelerate the optimization process and understand the advantage of using a large number of additional points, i.e. the gain of using a large number of additional boreholes to characterize the rock mass.

As result of this uni-objective optimization, Fig. 6 reports the optimal value of each objective function for every number of tested points. This graphical representation gives a more practical view of the differences in the objective function value when a small or a high number of additional points is considered. 


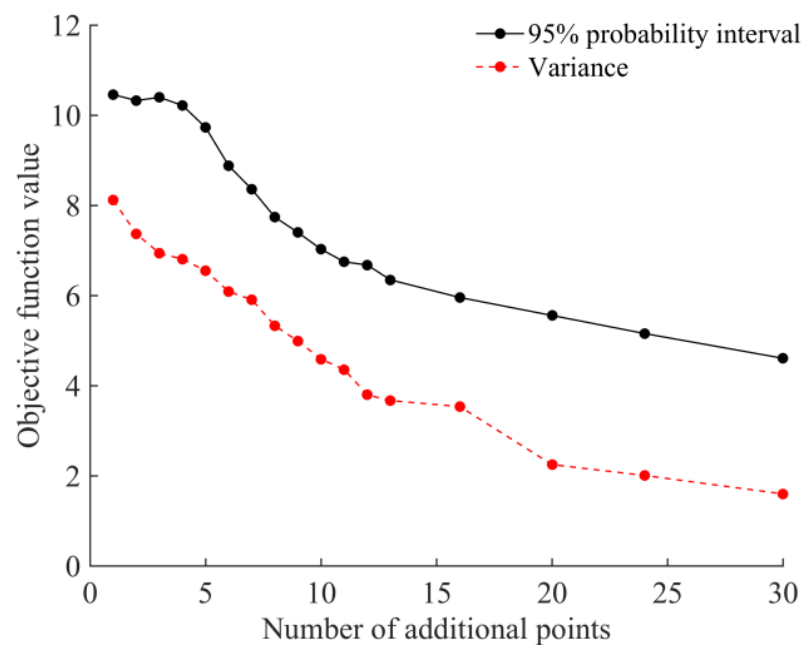

Fig. 6. Convergence study for the number of additional points considering the average width of $95 \%$ probability intervals (black line) and the average variance (red line).

It is possible to observe, as expected, that both objective functions values decrease as the number of additional points increases. However, this decrease is not constant. In what concerns the variance objective function, the decrease is significant until 12 additional points and also when the number of points increases from 16 to 20 . After this value, the decrease in the objective function is modest. In relation to the probability interval objective function, a significant reduction in the value is only observed starting from 5 additional points and up to 13 . After this value the decrease rate is smaller.

Fig. 7 shows a $3 D$ representation with the optimized positions for 12 additional points at each objective function, along with the representation of the preliminary data (black points). There is some proximity between the points optimized with each objective function, however their spatial locations are different, meaning that both objective functions behave differently. Also, it is interesting to notice that some of the points are aligned, which allow their simultaneous characterization by performing non-vertical boreholes that reduce the execution costs. For this specific case, using the variance results, the number of additional boreholes to perform is not 12 , as the number of suggested points, but can be reduced for, approximately, 7 or 8 boreholes (Fig. 7a). Even so, in Fig. $7 b$ the number of needed boreholes is lower (5 to 6 boreholes). 


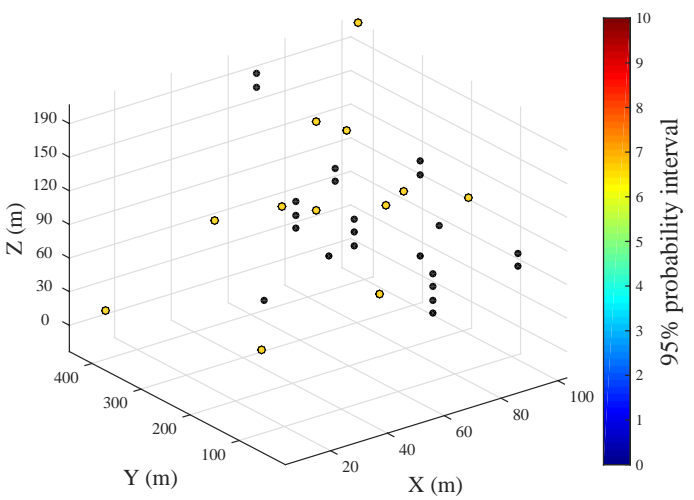

(a)

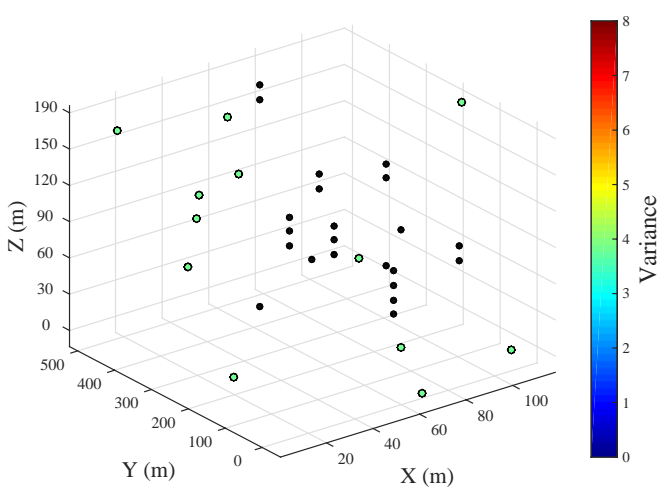

(b)

Fig. 7. $3 D$ location of 12 additional points with preliminary data in black points for: a) average width of $95 \%$ probability intervals; b) average variance.

Concerning the SA output parameters, for the specific case of 12 points, the final temperature is 0.08 for both objective functions and these functions are evaluated a total of 400 times. Also, the computational time spent in all the process is of 9 hours approximately.

In addition, Fig. 8 shows a $3 D$ graph that intends to represent the search block used for this study. It is possible to observe that the algorithm was able to search quite well within the block in order to find the optimal combination of 12 points.

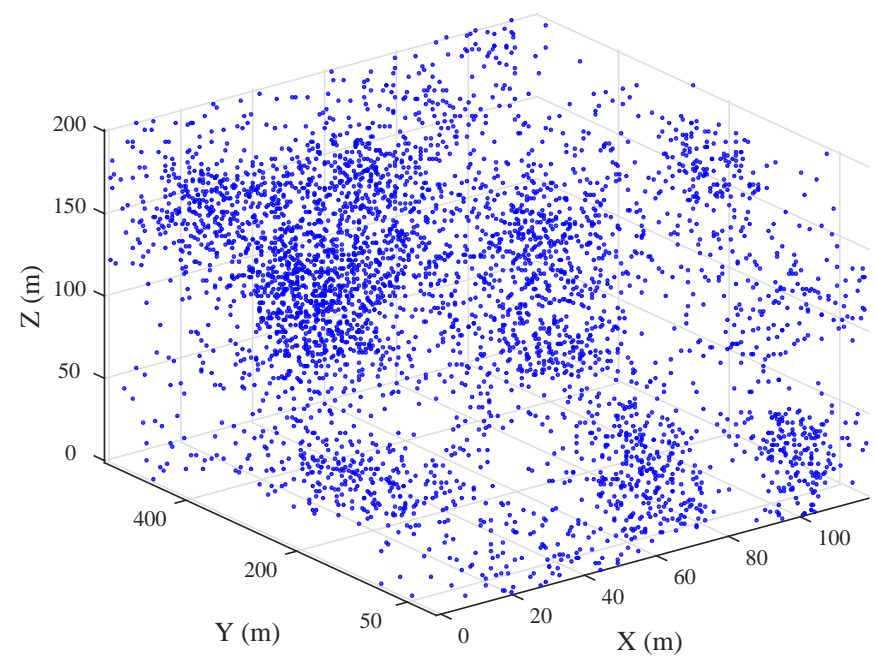

Fig. 8. 3D representation of the block used in the search for the Var function optimization when 12 points are added. 


\subsubsection{Point alignment optimization}

Following the same logic as for the isolated point optimization, the point alignment optimization also starts by adding one alignment of points composed by a total of 12 points $(n=12)$, then 5 alignments were added one by one. As a result, Fig. 9 presents the optimal value for each one of the objective functions for every alignment of points added to the preliminary data.

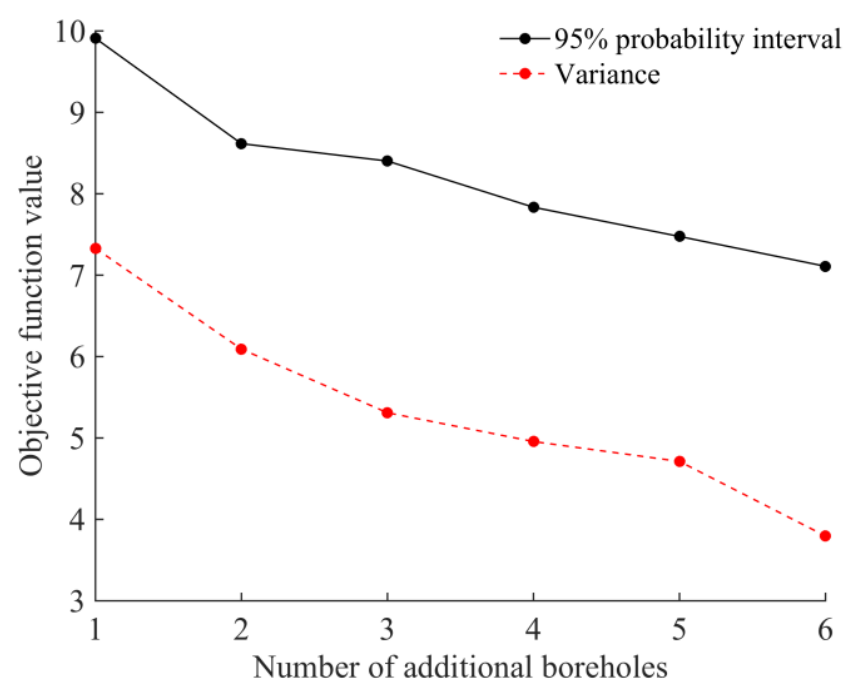

Fig. 9 Convergence study for the number of additional alignments considering the average width of 95\% probability intervals (black line) and the average variance (red line).

As observed in the isolated point optimization, both objective functions values decrease as the number of additional alignments increases. The decrease is more significant for the variance objective function when passing from 5 to 6 alignments, while the $95 \%$ probability function shows a more constant behavior.

Fig. 10 shows the $3 D$ maps with the optimized positions for 3 additional alignments at each objective function along with the representation of the preliminary data (black points). In this case and when compared to the isolated point optimization, one notices that the obtained alignments are located closer to the preliminary alignments; however, the spatial locations of the alignments for both objective functions are considerably different, showing, once again, that both functions behave distinctly. 


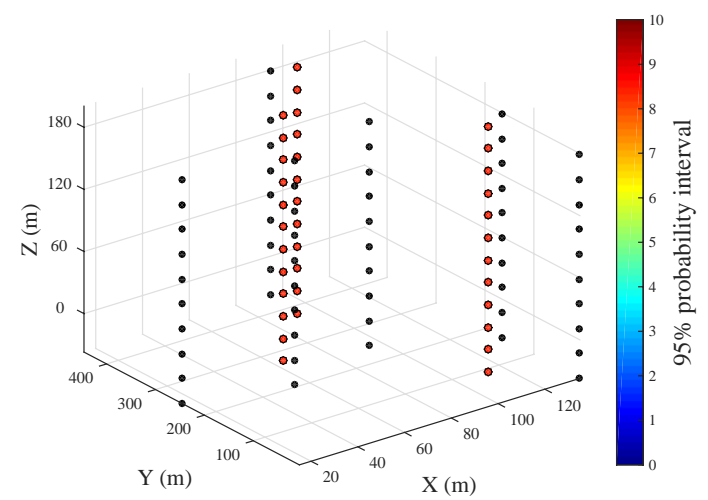

(a)

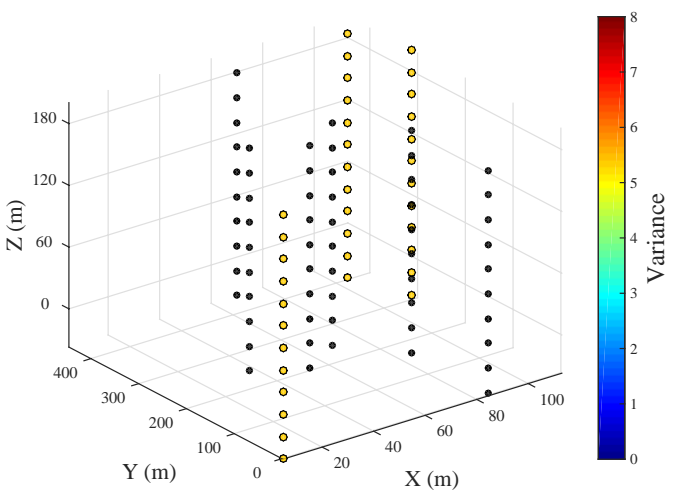

(b)

Fig. 10. 3D location of 3 additional point alignments with preliminary data in black points for: a) average width of $95 \%$ probability intervals; b) average variance.

\subsection{Multi-criteria results}

To provide an insight into the most influential objective function in the optimization results for each objective function, different weights values are assigned. These combinations of weights are presented in Table 4 along with the optimization results for both objective functions when 5 points $(n=5)$ are added to the 22 preliminary points (scenario with isolated points). This number of additional points is chosen based on the fact that a total of 5 additional boreholes can be sufficient to increase the quality of the geotechnical model (performing a large number of boreholes is not common in geotechnical works that are most often bounded to narrow budgets). This approach is also performed in the scenario with point alignments; however, considering the similarity of the results, the scenario with isolated points is chosen to be presented as an example.

Since trying a large number of weights can lead to a computational burden, a total of 11 most common combinations of weights are considered and represented in Table 4 by the test number, while the $\operatorname{Var}$ and $\overline{P I}_{95 \%}$ values are presented in their normalized scale. The representation of the solutions in the objective space is depicted in Fig. 11. 
Table 4 Optimization results for each combination of weights.

\begin{tabular}{ccccc}
\hline Test number & $\boldsymbol{W}_{\mathbf{1}}$ & $\boldsymbol{W}_{\mathbf{2}}$ & $\overline{\mathbf{P I}}_{\mathbf{9 5} \%}$ & $\boldsymbol{V a r}$ \\
\hline $\mathrm{T} 1$ & 0.00 & 1.00 & 0.85 & 0.69 \\
$\mathrm{~T} 2$ & 0.10 & 0.90 & 0.87 & 0.72 \\
$\mathrm{~T} 3$ & 0.20 & 0.80 & 0.86 & 0.72 \\
$\mathrm{~T} 4$ & 0.25 & 0.75 & 0.84 & 0.69 \\
T5 & 0.40 & 0.60 & 0.82 & 0.68 \\
T6 & 0.50 & 0.50 & 0.86 & 0.71 \\
T7 & 0.60 & 0.40 & 0.89 & 0.73 \\
T8 & 0.75 & 0.25 & 0.90 & 0.67 \\
T9 & 0.80 & 0.20 & 0.91 & 0.76 \\
T10 & 0.90 & 0.10 & 0.82 & 0.67 \\
T11 & 1.00 & 0.00 & 0.84 & 0.70 \\
\hline
\end{tabular}

In Fig. 11, the $X$-axis represents the $\overline{P I}_{95 \%}$ values obtained for each weighted test and the $Y$-axis, likewise, represents the $\operatorname{Var}$ values. As expected, one can observe that both objectives are not conflicting since the points represented in Fig. 11 result in a linear trend. In this sense, it is possible to detect that T5 and T10 result in the non-dominated points since the trade-off between the two objectives is softer, showing that the ideal solution should not exclude the variance criterion (T10). Also, T8 that valorizes the probability interval in $75 \%$ gives a worse solution (a solution far from the diagonal). This information confirms that the variance criterion, even for lower weight values, results in objective function values that are lower than $\overline{P I}_{95 \%}$, meaning that cannot be excluded of the optimization process and should be seen as the main objective function.

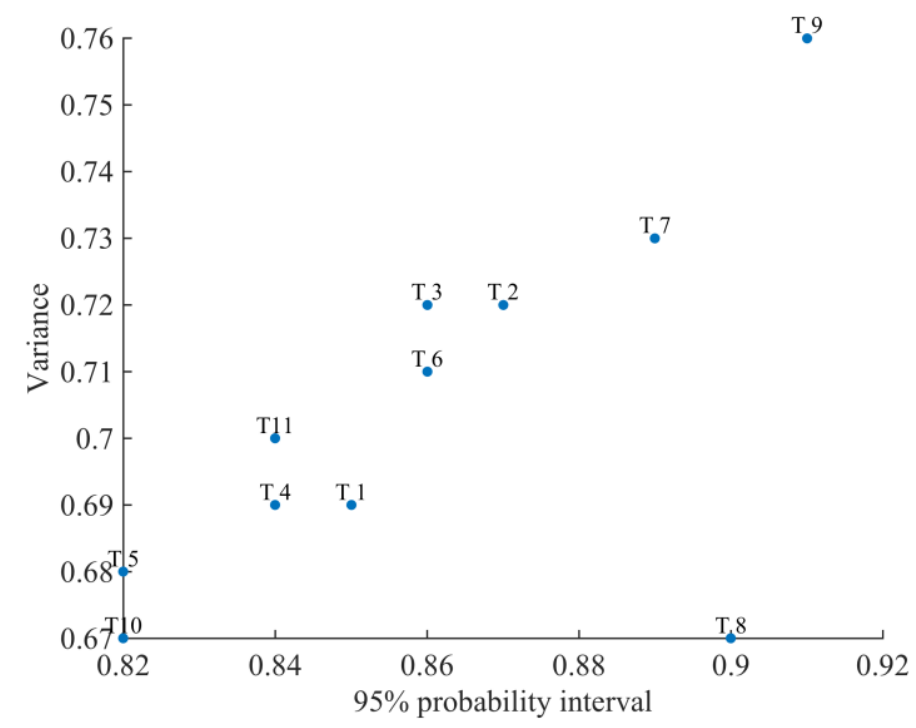

Fig. 11. Objective function values for each weight combination test. 
Furthermore, and in order to easily understand the contribution of each function in the decision of the number of boreholes to execute, Fig. 12 presents the performance of each objective function individually and the aggregation of the two objective functions in the multi-criteria approach, for each weight combination test. Throughout the graphical analysis it is possible to observe that, for the first five tests, where the variance objective weights are higher, the combination of the two objective functions (darkest line) shows the lowest values, confirming that the variance behaves as the best metric to use in the optimization process to decide the number of additional boreholes to be executed.

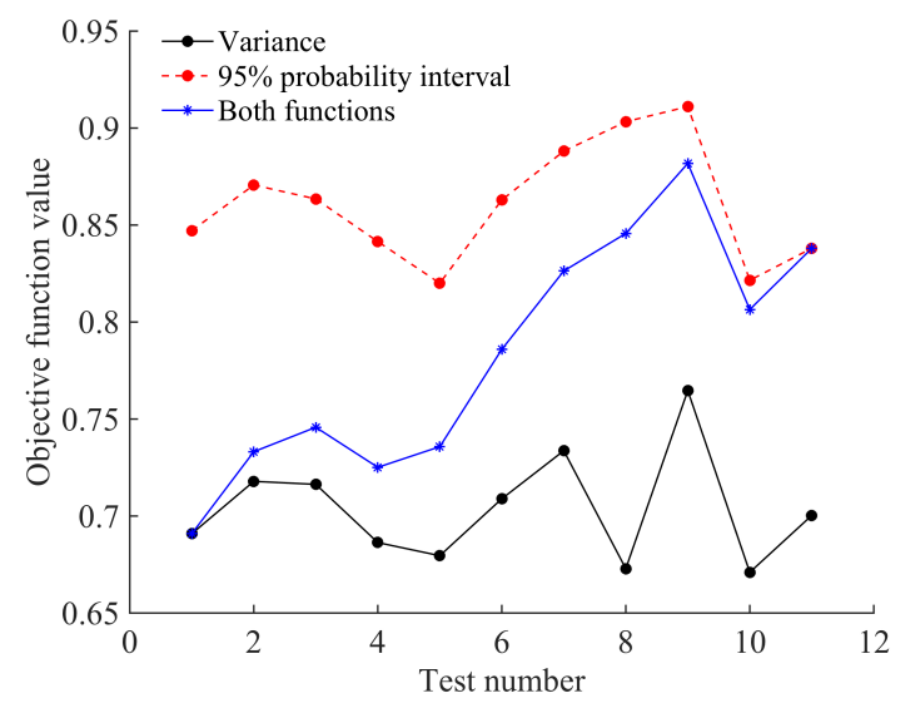

Fig. 12. Representation of the objective function values obtained in the multi-criteria approach.

\subsection{Discussion}

Both approaches provide an important insight into how optimizing the boreholes location increases the geotechnical detail of the rock mass and decreases the associated uncertainties. The uni-objective problem shows good results in giving the boreholes near-optimal locations according to two different objective functions. Fig. 6 displays the resulting curve between the variance value and the number of additional points tested, and it is possible to observe a higher downhill compared with the probability interval objective function. Also, it is possible to notice the differences between assuming the borehole as an isolated point or as a point alignment; however, they are not as significant as expected, once the total number of points used in the alignment optimization is almost the triple as the one used in the point optimization. In terms of values, the biggest difference is observed for Var function, where for one point versus 
one alignment the variance value is $10 \%$ lower for the last case, while the $\overline{P I}_{95 \%}$ shows a reduction of only 5\% compared with the results obtained from isolated point optimization.

In this regard, Fig. 12 shows that when a higher weight is given to the probability interval function (T7 to T11) the $\overline{P I}_{95 \%}$ value is, sometimes, higher and the variance is lower. This effect is called a trade-off (how much of a loss in one objective is one willing to sacrifice for a gain in another objective) and, in this case, the probability interval is the objective function that sacrifices more, i.e. minimizes slower when the goal is to give importance to the variance objective. Although, identifying this tradeoff may become difficult, once both functions are dimensionless (Marler and Arora, 2009). Nevertheless, this type of trade-off analysis can be helpful for the decisionmaker in the weight values definition and in choosing the best metric to achieve the optimization goals.

\section{CONCLUSIONS}

The goal of this work was to define a methodology to optimize boreholes plans commonly needed in geotechnical engineering. The proposed methodology combines two important methods: geostatistical conditional simulation, which results in the definition of the objective functions to measure the uncertainty at locations without data, and simulated annealing, used to perform the optimization by minimizing the defined objective functions. Two different objective functions are obtained and tested in this work: the average variance of the simulated values and the average width of the $95 \%$ probability intervals of the simulated values over a region of interest. Moreover, not only a uni-objective problem is solved, but also a multi-criteria approach is carried out using the weight sum method. The methodology was applied using real data from a deposit located in Chile and a different number of additional isolated points or point alignments (each alignment representing an additional borehole) were found in order to culminate in the best spatial location for each isolated point or point alignment.

Compared with the multi-criteria approach, the uni-objective approach presents the advantage of needing a lower computational time ( 9 to 10 hours while the multi-criteria approach requires $18 \mathrm{~h}$ to $22 \mathrm{~h}$ ), as the use of only one metric as an objective function 
simplifies the problem. On the other hand, the multi-criteria approach allows the decision-maker to give more importance to the metric (objective function) that is considered relevant, increasing the confidence in the optimization results.

Regarding the isolated point and point alignment optimizations, the results are very different, mainly because of the total number of initial points used in both cases: in isolated point optimization, a total of 22 points are used, while 62 points are used in point alignment optimization. In the latter case, the objective functions are not as low as expected, compared with the isolated point optimization. This fact comes to endorse that the $X Y$ location of the boreholes assumes, in this case, an important role in geotechnical prospection, although the point alignment optimization brings a more realistic output to represent boreholes.

Both optimization problems are formulated in order to apply the proposed methodology and show good results in finding the near-optimal locations for the additional boreholes. Hence, it is important to point out that a point-by-point study allows the decision-maker to understand the geotechnical gain balanced with the economic costs. For example, will performing five boreholes instead of four bring a significant gain in the rock mass characterization that justifies the difference in cost?

Therefore, one concludes that this methodology can contribute for a more rational approach in the formulation of prospection plans. Once the output is the measurement of the gain obtained from the addition of boreholes, including their spatial location, an indirect optimization of costs can be expected as a consequence. Also, it is worth mentioning that the output information of this methodology should be seen as complementary information to be paired with geological maps. One extra advantage associated with this methodology is the possibility to perform an updating in the information as the additional boreholes are executed.

In future works and in order to improve the proposed methodology it seems important to develop a multi-objective approach that aims at minimizing the number of boreholes and the associated costs. 


\section{ACKNOWLEDGMENTS}

This research is inserted in LNEC project named P2I-RockGeoStat and was partially funded by FCT (Fundação para a Ciência e Tecnologia, Portugal) in the scope of project PEst-UID/CEC/00319/2013, included in ISISE project UID/ECI/04029/2013 as well as the $\mathrm{PhD}$ grant SFRH/BD/89627/2012, and by the Chilean Commission for Scientific and Technological Research, through Project CONICYT PIA Anillo ACT1407.

\section{REFERENCES}

Aarts EHL, Korst JHM, 1989. Simulated Annealing and Boltzmann Machines. Wiley, Chichester.

Barton N, Lien R, Lunde J. Engineering classification of rock masses for the design of tunnel support. Rock Mechanics and Rock Engineering 1974; 6:189-236.

Bieniawski ZT, 1989. Engineering Rock Mass Classifications. Wiley, New York, USA, $272 \mathrm{p}$.

Brus DJ, Heuvelink GBM. Optimization of sample patterns for universal kriging of environmental variables. Geoderma 2007; 138:86-95.

Chilès JP, Delfiner P, 2012. Geostatistics modeling spatial uncertainty. John Wiley \& Sons, New York, 699p.

Emery X, Lantuéjoul C. TBSIM: A computer program for conditional simulation of three-dimensional Gaussian random fields via the turning bands method. Computer and Geosciences 2006;32(10):1615-1628.

Englund EJ, Heravi N. Phased sampling for soil remediation. Environmental and Ecological Statistics 1994; 1:247 - 263.

Hernandez J, Emery X. A geostatistical approach to optimize sampling designs for local forest inventories. Canadian Journal of Forest Research 2009; 39:1465 -1474.

Hoek E. Strength of rock and rock masses. ISRM News Journal 1994;2 (4):16. 
Kalamaras GS, Brino L, Carrieri G, Pline C, Grasso P. Application of multicriteria analysis to select the best highway alignment 2000. Tunnelling Underground Space Technol;15:415-420.

Kirkpatrick S, Gelatt CJr, Vecchi M. Optimization by simulated annealing. Science 1983;220 (4598):671-680.

Lin L, Fei C. The Simulated Annealing Algorithm Implemented by the MATLAB. International Journal of Computer Science Issues 2012;9(6):357-360.

Marchant BP, Lark RM. Adaptive sampling and reconnaissance surveys for geostatistical mapping of the soil. European Journal of Soil Science 2006; 57:831-845.

Marler RT, Arora JS. The weighted sum method for multi-objective optimization: new insights. Structures Multidiscipline Optimization 2009; 41:853-862.

McBratney AB., Webster R, Burgess TM. The design of optimal sampling schemes for local estimation and mapping of regionalized variables. Computers \& Geosciences $1981 ; 7: 331-334$.

Olea R. Sampling Design Optimization for Spatial Functions. Mathematical Geology $1984 ; 16(4): 369-392$.

Pinheiro M, Emery X, Miranda T, Vallejos J. Truncated Gaussian Simulation to Map the Spatial Heterogeneity of Rock Mass Rating, Rock Mechanics and Rock Engineering $2016 b ; 1-6$.

Pinheiro M, Vallejos J, Miranda T, Emery X. Geostatistical simulation to map the spatial heterogeneity of geomechanical parameters: A case study with rock mass rating. Engineering Geology 2016a; 205:93-103.

Scheck D, Chou DR. Optimum locations for exploratory drill holes. International Journal of Mining Engineering 1983; 1(4): 343-355.

Soltani S, Hezarkhani A. Additional exploratory boreholes optimization based on threedimensional model of ore deposit. Archives of Mining Science 2009; 54(3):495-506. 
Soltani S, Hezarkhani A, Erhan Tercan A and Karimi, B. Use of genetic algorithm in optimally locating additional drill holes. Journal of Mining Science 2011; 47(1):62-72.

Soltani S, Hezarkhani A. A simulated annealing-based algorithm to locate additional drillholes for maximizing the realistic value of information. Natural Resources Research 2013a;22(3).

Soltani S, Hezarkhani A. Proposed algorithm for optimization of directional additional exploratory drill holes and computer coding. Arabian Journal of Geosciences 2013b; $6(2): 455-462$.

Van Groenigen JW, Siderius W, Stein A. Constrained optimisation of soil sampling for minimisation of the kriging variance. Geoderma 1999; 87:239-259.

Yang XS, 2010. Engineering Optimization: An Introduction with Metaheuristic Applications. John Wiley \& Sons, NJ: University of Cambridge, 347 p.

Zadeh LA. Optimality and non-scalar-valued performance criteria. IEEE Trans Automat Contr AC 1963;8:59-60. 